\title{
RXTE determination of the intermediate polar status of XSS J00564+4548, IGR J17195-4100, and XSS J12270-4859
}

\author{
O. W. Butters ${ }^{1}$, A. J. Norton ${ }^{1}$, P. Hakala ${ }^{2}$, K. Mukai ${ }^{3}$, and E. J. Barlow ${ }^{1}$ \\ 1 Department of Physics and Astronomy, The Open University, Walton Hall, Milton Keynes MK7 6AA, UK \\ e-mail: o.w.butters@open.ac.uk \\ 2 Tuorla Observatory, University of Turku, 21500 Piikkiö, Finland \\ 3 CRESST and X-ray Astrophysics Laboratory NASA/GSFC, Greenbelt, MD 20771, USA
}

Received 10 April 2008 / Accepted 24 May 2008

\begin{abstract}
Aims. We determine the nature of the intermediate polar candidates XSS J00564+4548, IGR J17195-4100, and XSS J12270-4859. Methods. Pointed RXTE observations searched for intermediate polar characteristics in these candidate systems. Results. XSS J00564+4548 exhibits a period of $465.68 \pm 0.07 \mathrm{~s}$, which we interpret as the spin period, an energy dependent modulation depth, and a spectrum that is fit by a $22 \mathrm{keV}$ photoelectrically absorbed bremsstrahlung with an iron line profile. IGR J17195-4100 shows several candidate periodicities and a spectrum that is fit by a power law with an iron line. XSS J12270-4859 exhibits a candidate spin period of $859.57 \pm 0.64 \mathrm{~s}$ and a spectrum that is fit by a power law with no evidence of an iron line. Conclusions. XSS J00564+4548 is confirmed to be an intermediate polar. IGR J17195-4100 and XSS J12270-4859 both show some properties of intermediate polars, but cannot be confirmed as definite members of the class here.
\end{abstract}

Key words. binaries: close - stars: novae, cataclysmic variables - X-rays: binaries

\section{Introduction to magnetic cataclysmic variables}

Intermediate polars (IPs) belong to the class of systems known as cataclysmic variables (CVs). They occupy the phase space, in terms of magnetic field strength, between the polars and the non-magnetic CVs. This intermediate strength magnetic field alters the accretion flow from the main sequence donor star to the white dwarf (WD). Eventually, most of the accreting material is channelled to accretion curtains above the WD magnetic poles. The temperature and density of this region causes the emission of bremsstrahlung radiation, which varies at the spin period of the WD. It is this variation that most consider to be the defining characteristics of IPs. For a review of IPs see e.g. Warner (1995). There are at least 30 confirmed IPs ${ }^{1}$. Ramsay et al. (2008), however, have recently pointed out that the commonly used criteria to certify CVs as IPs may be too restrictive. It is possible that many of the 84 candidates $^{1}$ are indeed IPs, and if classes such as SW Sex systems are in fact IPs then the true number may be several hundred.

In recent years there have been 16 IPs found to emit in the hard X-ray/soft gamma-ray part of the spectrum, with the INTEGRAL/IBIS survey (Barlow et al. 2006; Bird et al. 2007). With this in mind we have embarked on a campaign to observe some hard X-ray sources and determine their credentials as potential IPs. In the first paper in this campaign, SWIFT J0732.51331 was confirmed as an IP (Butters et al. 2007). Here the results of pointed RXTE observations of XSS J00564+4548 (hereafter J0056), IGR J17195-4100 (hereafter J1719) and XSS J12270-4859 (hereafter J1227) are presented.

1 asd.gsfc.nasa.gov/Koji.Mukai/iphome/iphome.html as at 30/04/08.

\section{Previous observations}

J0056 was associated with the ROSAT source 1RXS J005528.0+461143, and catalogued as an unidentified object in the RXTE all sky survey (Revnivtsev et al. 2004). It was found to have a count rate of $0.71 \pm 0.04 \mathrm{ct} \mathrm{s}^{-1} \mathrm{PCU}^{-1}$ in the 3-8 keV energy band and a photon index of $1.77 \pm 0.23$. Analysis by Bikmaev et al. (2006) using SWIFT/XRT archive data revealed two X-ray sources in the ROSAT error circle. One source was present at low energy, which they presumed to be a chromospherically active star. The other source showed a typical spectrum of a $\mathrm{CV}$, with an emission feature close to $6.7 \mathrm{keV}$. Bikmaev et al. (2006) also carried out optical observations with the $1.5 \mathrm{~m}$ Russian-Turkish Telescope. Their photometric data indicated a period of approximately $480 \mathrm{~s}$ to be present.

J1719 was detected as an INTEGRAL object by Bird et al. (2004); Pandey et al. (2006) found radio galaxies coincident with its error circle and suggested it was extragalactic. Tomsick et al. (2006) confirmed a tentative association of J1719 with the softer X-ray target 1RXS J171935.6-410054 using pointed Chandra data. They also reported variability of J1719 in the $0.3-10 \mathrm{keV}$ band and a flux of $2.5_{-0.4}^{+0.9} \times 10^{-11} \mathrm{erg} \mathrm{cm}^{-2} \mathrm{~s}^{-1}$. In calculating this flux they used a power law model and a galactic column density of $0.77 \times 10^{22} \mathrm{~cm}^{-2}$ (derived from Dickey \& Lockman 1990). Tomsick et al. (2006) also reported the spectral properties of J1719 using public INTEGRAL data, finding a flux of $1.9 \times 10^{-11} \mathrm{erg} \mathrm{cm}^{-2} \mathrm{~s}^{-1}$ in the $20-50 \mathrm{keV}$ energy band. Masetti et al. (2006) classified J1719 as a CV based upon its optical spectrum, they also speculated that it may be an IP.

J1227 was found in the RXTE all sky survey (Revnivtsev et al. 2004). It was classified as a CV and suggested to be an IP 
by Masetti et al. (2006), using optical spectroscopy. Bird et al. (2007) later found J1227 to be an INTEGRAL source.

\section{Observations and data reduction}

Data were obtained from the RXTE satellite (Bradt et al. 1993) with the PCA instrument.

In each case initial data reduction was done with the standard FTOOLS, and the flux was normalised according to the number of correctly functioning PCUs. For the lightcurve analysis PCUs 2, 3, and 4 were used; whilst for the spectral analysis only PCU 2 was used. Only the top layer of each PCU was included in the measurements and the time resolution of the data was $16 \mathrm{~s}$. Background subtracted light curves were constructed in four energy bands: 2-4 keV, 4-6 keV, 6-10 keV and 10-20 keV, as well as a combined 2-10 keV band for maximum signal-to-noise.

In the presence of white noise in the data, the power values in the power spectrum are expected to follow an exponential distribution. However, any correlated noise e.g. red noise, will mean the distribution becomes frequency dependent. This makes estimating the significance limits in the power spectra non-trivial. As accreting systems usually show flickering in their lightcurves, it is feasible to believe that there may be a significant red noise component in the data. In order to take this into account in the analysis, the technique introduced in Hakala et al. (2004) was used. The data were equally spaced (apart from the large gaps in between different orbits), so the red noise component was modelled by fitting a second order autoregressive process model to the lightcurves. This model was then used to generate 50000 synthetic lightcurves with similar red and white noise properties, as well as observing window, to the original datasets. The $95.2 \%, 99.72 \%$ and $99.954 \%$ (2, 3 and $4 \sigma$ respectively) significance limits (as a function of frequency) were then calculated.

To estimate the error on the measured periods we folded the raw data at the period found from the period analysis. We then fitted a curve to this folded data. This curve (repeated over the whole data set) was then subtracted from the raw data leaving residual values. These were then shuffled and added to the fitted curve, yielding a new synthetic raw data set. This synthetic data was then analysed as before. This whole process was repeated $\sim 200$ times and the resulting periods were then used to calculate a standard deviation of periods, which was then used as the error estimate.

A mean X-ray spectrum was also extracted for each source, and two spectral models applied to find the best fit, using the XSPEC package. The models considered were a photoelectrically absorbed single temperature bremsstrahlung with a Gaussian at the iron line emission energy (model A), and a photoelectrically absorbed power law with a similar Gaussian (model B).

\subsection{XSS J00564+4548}

J0056 was observed over two consecutive days (see Table 1). The total good time on target (37800 s) comprised fourteen approximately equal segments of one satellite orbit each. In the $2-10 \mathrm{keV}$ energy band the raw count rate varied between 3.9 and $9.1 \mathrm{ct} \mathrm{s}^{-1} \mathrm{PCU}^{-1}$. The background count rate, generated from the calibration files, varied between 2.9 and $4.1 \mathrm{ct} \mathrm{s}^{-1} \mathrm{PCU}^{-1}$.

A significant $(>4 \sigma)$ peak was present in the periodogram at $\sim 185$ cycles day $^{-1}$ in the $2-10 \mathrm{keV}$ energy band (see Fig. 1). Analysis of the peak gave a pulsation period of $465.68 \pm 0.07 \mathrm{~s}$. The data were then folded in each energy band at this period,
Table 1. Observing log.

\begin{tabular}{ccccc}
\hline \hline Target & $\begin{array}{c}\text { Start time } \\
\text { (UTC) }\end{array}$ & $\begin{array}{c}\text { End time } \\
\text { (UTC) }\end{array}$ & $\begin{array}{c}\text { Time } \\
\text { on target } \\
(\mathrm{s})\end{array}$ & $\begin{array}{c}\text { Good } \\
\text { time }^{a} \\
(\mathrm{~s})\end{array}$ \\
\hline J0056 & $05: 2720 / 12 / 07$ & $00: 3122 / 12 / 07$ & 84672 & 37800 \\
J1719 & $18: 4507 / 01 / 08$ & $11: 1609 / 01 / 08$ & 69636 & 35936 \\
J1227 & $16: 1328 / 11 / 07$ & $16: 2029 / 11 / 07$ & 58183 & 26814 \\
\hline
\end{tabular}

${ }^{a}$ Good time is defined as the time that met our selection criteria.

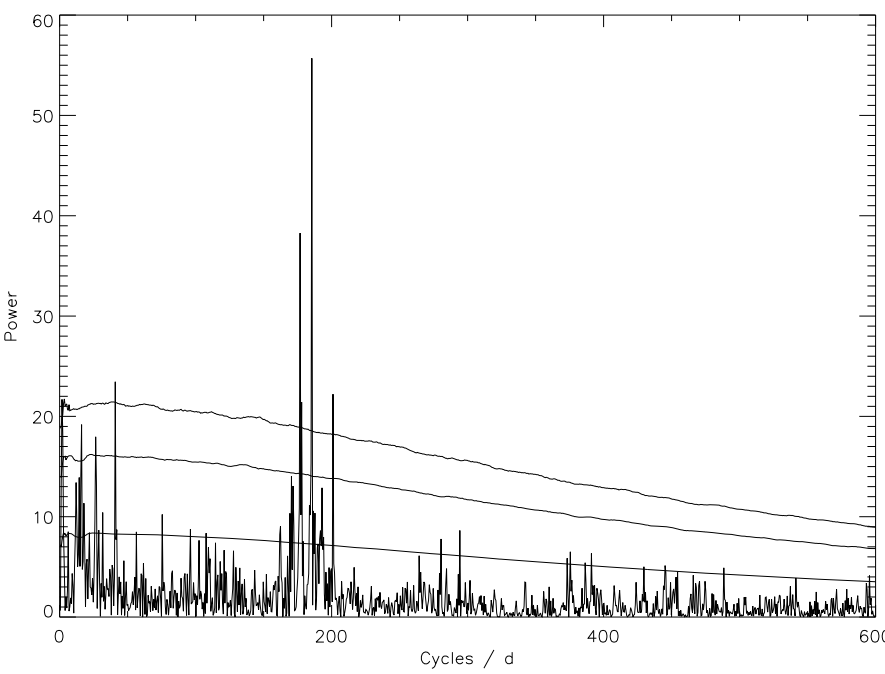

Fig. 1. 2-10 keV periodogram of J0056. Three significance levels, 95.2, 99.7 and $99.954 \%$ (2, 3 and $4 \sigma$ respectively), are superimposed.

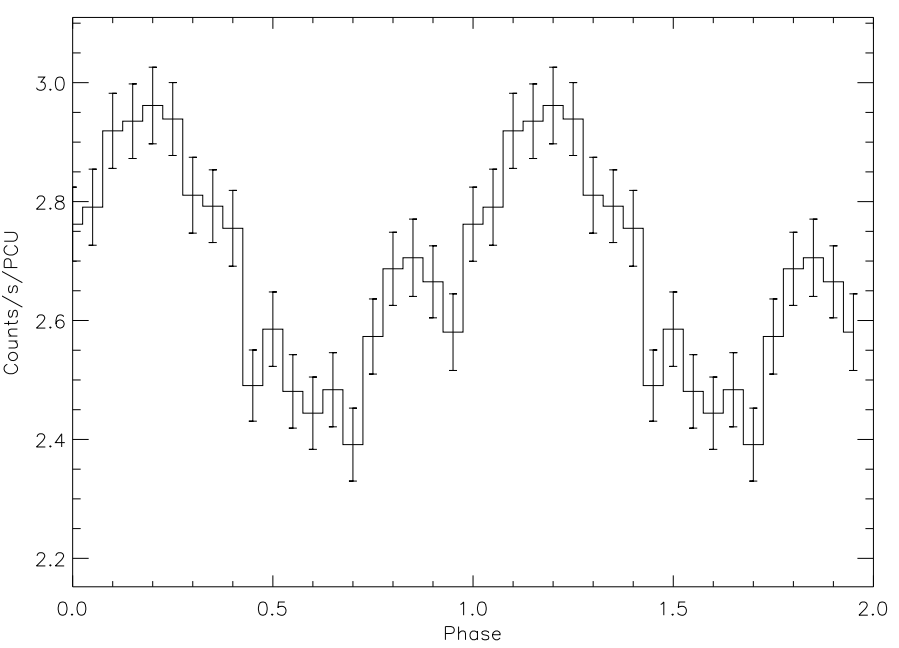

Fig. 2. 2-10 keV lightcurve of J0056 folded at the $465.68 \mathrm{~s}$ pulse period with an arbitrary zero point. Two cycles are shown for clarity.

Fig. 2 shows the result of the $2-10 \mathrm{keV}$ energy band. In each energy band a sinusoid was fitted to the folded data to estimate the modulation depth of the variation (see Table 2). There is a clear decreasing trend in the modulation depth with increasing energy. Clustered around the 185 cycles day $^{-1}$ peak were a series of smaller peaks, spaced apart by $\sim 8$ cycles day $^{-1}$, the largest of which was at $489.0 \pm 0.7 \mathrm{~s}$. There was also one other peak detected at above the $4 \sigma$ level at $\sim 41$ cycles day $^{-1}(2109 \mathrm{~s})$.

The best spectral fit was a simple photoelectrically absorbed bremsstrahlung model with a Gaussian added. This fit had the parameters $k T=22 \pm 2 \mathrm{keV}, n_{\mathrm{H}}=(0.6 \pm 0.4) \times 10^{22} \mathrm{~cm}^{-2}$ and a Gaussian at $6.5 \pm 0.1 \mathrm{keV}$ with a width of $0.3 \pm 0.1 \mathrm{keV}$, 
Table 2. Modulation depths of the pulse profile in different energy bands. Modulation depth is defined here as the semi-amplitude of a fitted sinusoid compared to the fitted mean.

\begin{tabular}{ccccccc}
\hline \hline & \multicolumn{2}{c}{$\mathrm{J}^{2}$} & \multicolumn{2}{c}{$\mathrm{J}_{1716^{b}}$} & \multicolumn{2}{c}{$\mathrm{J}^{b} 27^{c}$} \\
$\begin{array}{c}\text { Energy band } \\
(\mathrm{keV})\end{array}$ & $\begin{array}{c}\text { Modulation depth } \\
(\%)\end{array}$ & $\begin{array}{c}\text { Fitted mean } \\
\left(\mathrm{ct} \mathrm{s}^{-1} \mathrm{PCU}^{-1}\right)\end{array}$ & $\begin{array}{c}\text { Modulation depth } \\
(\%)\end{array}$ & $\begin{array}{c}\text { Fitted mean } \\
\left(\mathrm{ct} \mathrm{s}^{-1} \mathrm{PCU}^{-1}\right)\end{array}$ & $\begin{array}{c}\text { Modulation depth } \\
(\%)\end{array}$ & $\begin{array}{c}\text { Fitted mean } \\
\left(\mathrm{ct} \mathrm{s}^{-1} \mathrm{PCU}^{-1}\right)\end{array}$ \\
\hline $2-10$ & $8 \pm 1$ & 2.69 & $5 \pm 1$ & 4.22 & $26 \pm 2$ & 1.29 \\
$2-4$ & $14 \pm 2$ & 0.59 & $4 \pm 1$ & 1.01 & $27 \pm 3$ & 0.37 \\
$4-6$ & $9 \pm 1$ & 0.93 & $4 \pm 1$ & 1.45 & $25 \pm 3$ & 0.45 \\
$6-10$ & $5 \pm 1$ & 1.17 & $6 \pm 1$ & 1.75 & $27 \pm 3$ & 0.47 \\
$10-20$ & $8 \pm 3$ & 0.60 & $5 \pm 2$ & 0.72 & $28 \pm 7$ & 0.22 \\
\hline
\end{tabular}

${ }^{a}$ Folded at the $465.68 \mathrm{~s}$ period; ${ }^{b}$ folded at the possible period of $1842.4 \mathrm{~s} ;{ }^{c}$ folded at the $859.57 \mathrm{~s}$ period.

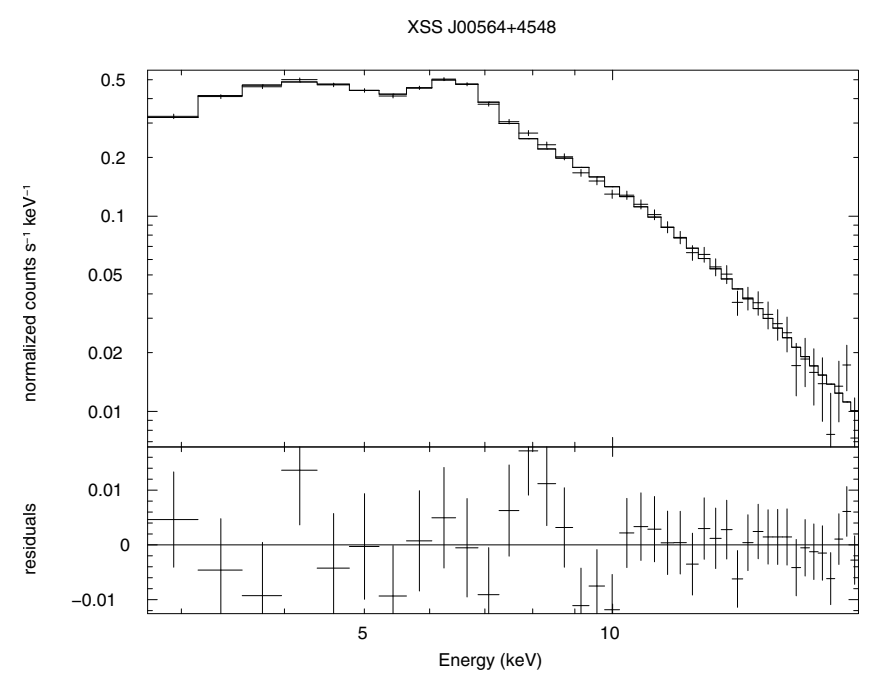

Fig. 3. 2.5-20 keV mean spectrum of J0056 fitted with a photoelectrically absorbed bremsstrahlung plus iron line profile.

which was interpreted as a iron feature $\left(\chi_{\text {reduced }}^{2}=0.8\right)$, as shown in Fig. 3 and summarised in Table 3. The table also shows the Galactic column density to the object as derived from the $\operatorname{HEASARC} n_{\mathrm{H}}$ tool $^{2}$.

\subsection{IGR J17195-4100}

Data were taken over two consecutive days (see Table 1). The total good time on target (35936 s) was split over twelve approximately equal segments. The raw target flux varied from $5.4-11.3 \mathrm{ct} \mathrm{s}^{-1} \mathrm{PCU}^{-1}$ and the generated background varied from $2.8-3.9 \mathrm{ct} \mathrm{s}^{-1} \mathrm{PCU}^{-1}$.

The periodogram of $\mathrm{J} 1719$ had six potential periods that were over $4 \sigma$, see Fig. 4 . To discount any artifacts arising from the windowing of the raw data we also used the CLEAN algorithm of Lehto (1997). This was a necessary step as the raw data was rather fragmented. This iteratively deconvolved the window function from any signals present in the lightcurve itself. The four peaks between 8 and 22 cycles day ${ }^{-1}$ were found to have a much lower significance in the CLEANed analysis and were thus discounted as an artifact of the windowing. Both remaining peaks above the $4 \sigma$ level $(1842.4 \pm 1.5 \mathrm{~s}$ and $2645.0 \pm 4.0 \mathrm{~s})$ were equally viable periods. We selected the $1842.4 \mathrm{~s}$ period to fold the data at, but we stress that the other period was an equally likely candidate period, see Fig. 5. Folding the data in each energy band at this period showed that the modulation depth is

${ }^{2}$ http://heasarc.nasa.gov/cgi-bin/Tools/w3nh/w3nh.pl

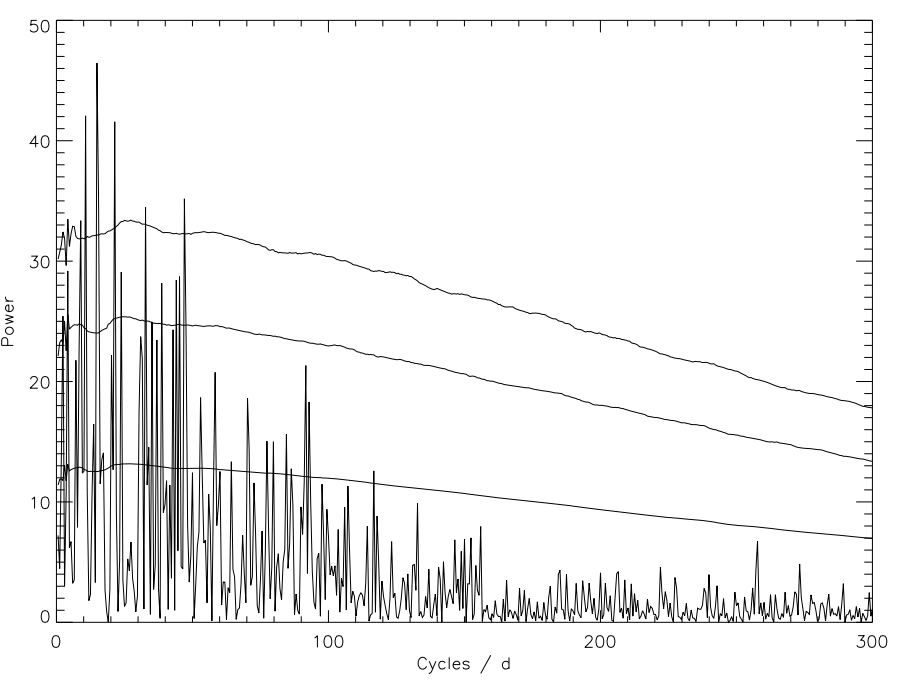

Fig. 4. 2-10 keV periodogram of J1719. Three significance levels, 95.2, 99.7 and $99.954 \%$ (2, 3 and $4 \sigma$ respectively), are superimposed.

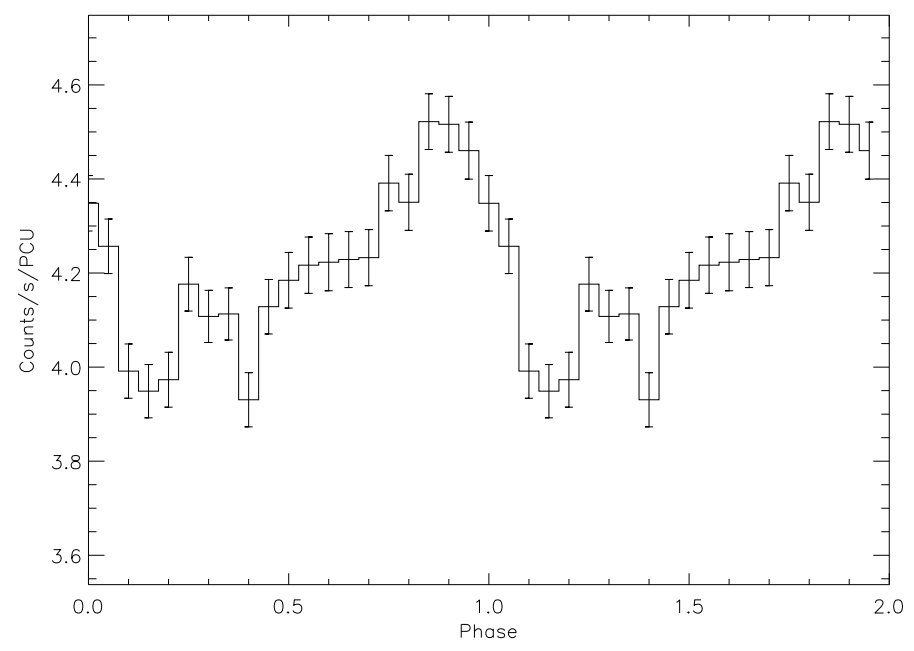

Fig. 5. 2-10 keV folded lightcurve of J1719. Folded at $1842.4 \mathrm{~s}$ with an arbitrary zero point. Two periods are shown for clarity.

constant across them all (see Table 2). We also note that there is a further peak (at just below $3 \sigma$ significance) at $941 \mathrm{~s}$, whose period is close to half that of the $1842.4 \mathrm{~s}$ candidate period, and may therefore represent a first harmonic.

Spectral analysis showed the presence of an iron line in a photoelectrically absorbed bremsstrahlung profile, however the fit was poor with $\chi_{\text {reduced }}^{2}=3.0$. A better fit was achieved with a power law model as shown in Fig. 6 and Table 3, however 
Table 3. Spectral fits.

\begin{tabular}{ccccccccccc}
\hline \hline Target & $\begin{array}{c}n_{\mathrm{H}}(\mathrm{Galactic}) \\
\left(\times 10^{22} \mathrm{~cm}^{-2}\right)\end{array}$ & Model & $\begin{array}{c}n_{\mathrm{H}} \\
\left(\times 10^{22} \mathrm{~cm}^{-2}\right)\end{array}$ & $\begin{array}{c}k T \\
(\mathrm{keV})\end{array}$ & $\Gamma$ & $\begin{array}{c}\mathrm{Fe} \\
(\mathrm{keV})\end{array}$ & $\begin{array}{c}\sigma_{\mathrm{Fe}} \\
(\mathrm{keV})\end{array}$ & $\begin{array}{c}E W \\
(\mathrm{keV})\end{array}$ & $\begin{array}{l}\chi_{\text {reduced }}^{2} \\
\left(\times 10^{-11} \mathrm{erg} \mathrm{cm}^{-2} \mathrm{~s}^{-1}\right)\end{array}$ \\
\hline J0056 & 0.1 & $\mathrm{~A}$ & $0.6 \pm 0.3$ & $22 \pm 3$ & - & $6.5 \pm 0.1$ & $0.3 \pm 0.1$ & 0.8 & 0.8 & \\
J0056 & 0.1 & $\mathrm{~B}$ & $1.9 \pm 0.7$ & - & $1.7 \pm 0.1$ & $6.5 \pm 0.1$ & $0.3 \pm 0.1$ & 0.9 & 1.1 & 2.8 \\
J1719 & 0.7 & $\mathrm{~A}$ & $0.7 \pm 0.1$ & $17 \pm 1$ & - & $6.5 \pm 0.1$ & $0.1 \pm 0.1$ & 0.5 & 3.0 & 4.4 \\
J1719 & 0.7 & $\mathrm{~B}$ & $0.7^{a}$ & - & $1.8 \pm 0.1$ & $6.5 \pm 0.1$ & $0.3 \pm 0.1$ & 0.7 & 1.1 & \\
J1227 & 0.1 & $\mathrm{~A}$ & $0.1^{a}$ & $14 \pm 1$ & - & $6.5^{\mathrm{b}}$ & $0.1^{\mathrm{b}}$ & $<0.08$ & 1.3 & 4.7 \\
$\mathrm{~J} 1227$ & 0.1 & $\mathrm{~B}$ & $0.1^{a}$ & - & $1.8 \pm 0.1$ & $6.5^{b}$ & $0.1^{b}$ & $<0.17$ & 0.8 & 1.5 \\
\hline
\end{tabular}

${ }^{a}$ Pegged to a lower limit of this value to reflect the Galactic column density; ${ }^{b}$ no error as this value was imposed. See notes in the text.

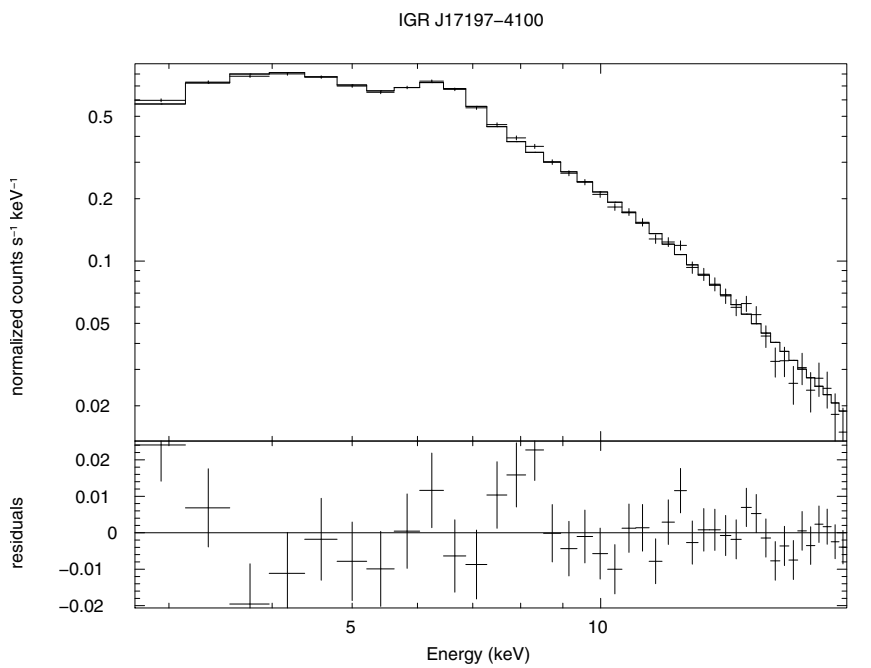

Fig. 6. 2.5-20 keV mean spectrum of J1719 fitted with a photoelectrically absorbed power law plus iron line profile.

this fit had the column density pegged to a lower limit of $0.7 \times$ $10^{22} \mathrm{~cm}^{-2}$ to reflect the galactic column density.

\subsection{XSS J12270-4859}

Data were collected over the course of just over one day (see Table 1). Total good time on target (26814 s) was split over nine segments. The raw target count rate varied between $2.4-10.9 \mathrm{ct} \mathrm{s}^{-1} \mathrm{PCU}^{-1}$, the generated background count rate varied between $2.8-3.9 \mathrm{ct} \mathrm{s}^{-1} \mathrm{PCU}^{-1}$.

Analysis of the lightcurve showed significant $(>4 \sigma)$ structure at $\sim 100$ cycles day $^{-1}$ (see Fig. 7 ). The peak of this structure was at $859.57 \pm 0.64 \mathrm{~s}$. Folding the data at this period showed a clear modulation in the $2-10 \mathrm{keV}$ energy band (see Fig. 8), with approximately the same percentage depth in each energy band (see Table 2). There was also a peak at approximately one cycle day ${ }^{-1}$ in the periodogram; we discounted this peak as it was of the order of the length of the observing run, and was probably a feature of the window function.

In fitting the spectrum, the column density was again pegged to the lower limit of the galactic column density for both the models. The best fit was the power law model, giving $\chi_{\text {reduced }}^{2}=$ 0.8 (see Table 3). There is no significant sign of an excess at the iron line energy (see Fig. 9). A Gaussian was fitted to the expected position of the iron emission feature, but in each case only a small upper limit to the equivalent width was found $(<0.08$ and $<0.17 \mathrm{keV}$ for models A and B respectively).

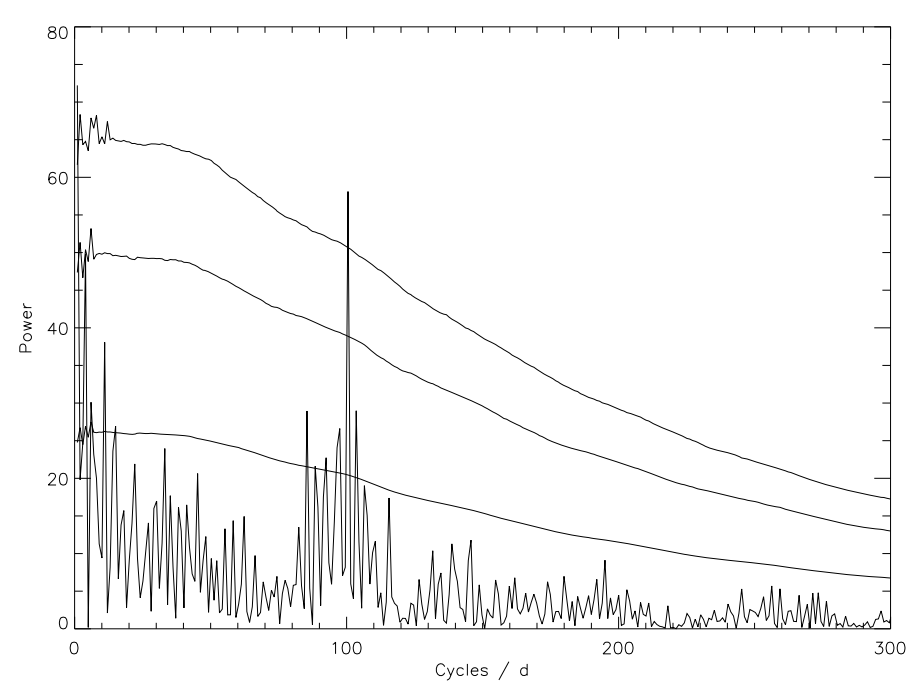

Fig. 7. 2-10 keV periodogram of J1227. Three significance levels, 95.2, 99.7 and $99.954 \%$ (2, 3 and $4 \sigma$ respectively), are superimposed.

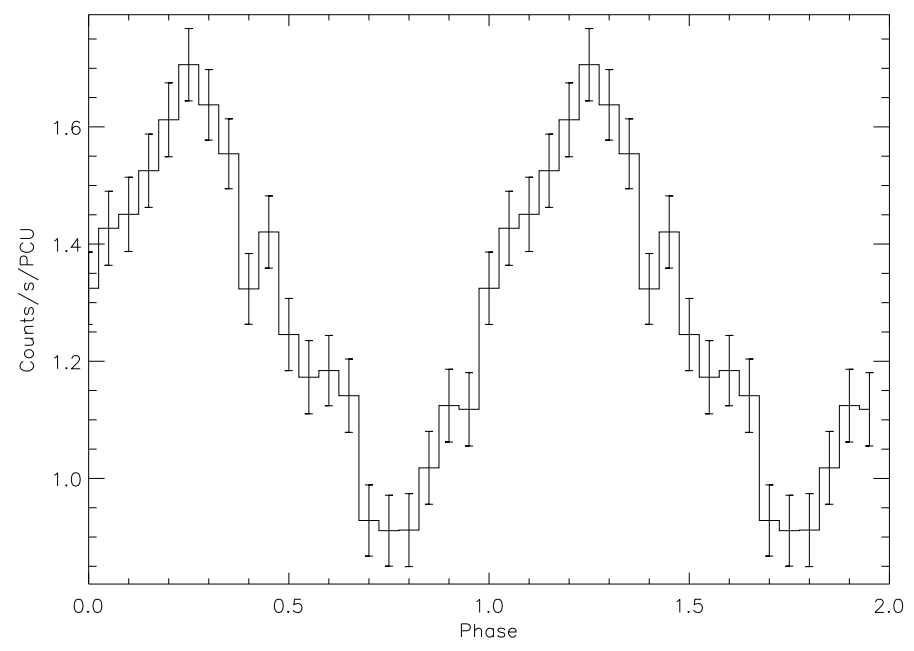

Fig. 8. 2-10 keV lightcurve of J1227 folded at the $859.57 \mathrm{~s}$ pulse period with an arbitrary zero point. Two cycles are shown for clarity.

\section{Discussion}

\subsection{XSS J00564+4548}

We interpret the period found here $(465.68 \pm 0.07 \mathrm{~s})$ as the spin period of the WD in J0056. Bikmaev et al. (2006) gave an approximate value of $480 \mathrm{~s}$ from their analysis. To obtain an estimate of the error in their period we consider the FWHM of their Lomb-Scargle plot, which gives $480 \pm 20 \mathrm{~s}$. Our period determination therefore is in agreement with their optical data. If 


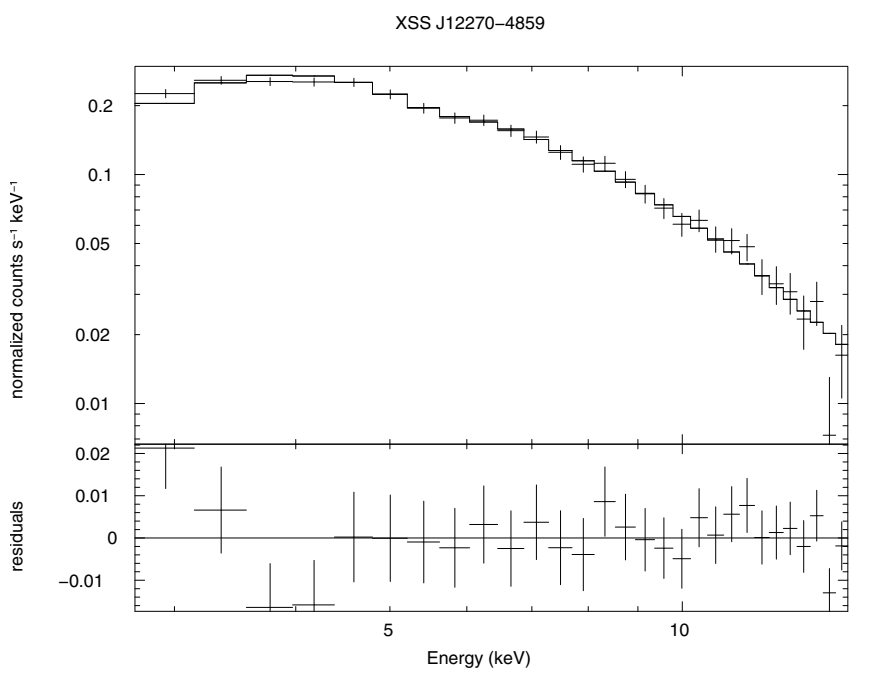

Fig. 9. 2-15 keV mean spectrum of J1227 fitted with a photoelectrically absorbed power law plus Gaussian.

we interpret the second strongest peak in our periodogram as a beat period, this implies the orbital period must be $\sim 2.7 \mathrm{~h}$. This places it in the 2-3 h CV period gap, but we note that there are now several CVs in this range and some $\mathrm{mCVs}$ too. This interpretation would also explain the cluster of peaks around the spin period as being various harmonics of the beat period. We are unsure of the origin of the 41 cycles day $^{-1}$ peak as it is too short to be interpreted as an orbital period of a typical IP. The energy dependant modulation depth of the folded lightcurves is common among IPs and indicates an accretion column absorbing structure (Norton \& Watson 1989). We also note that the pulse profile of J0056 is very similar in shape to that of FO Aqr (Beardmore et al. 1998).

The spectral fits indicate that the emission process is more likely to be a photoelectrically absorbed single temperature bremsstrahlung process rather than a power law, as is common in IPs. The power law spectral fit is however in agreement with Revnivtsev et al. (2004). The Gaussian at $6.5 \mathrm{keV}$ is identifiable with an iron feature which is also a common aspect of IPs, and agrees with the feature found by Bikmaev et al. (2006).

The ROSAT bright source catalogue has no other sources in the RXTE PCA field of view of J0056. Bikmaev et al. (2006) showed that there was a source in the error circle of ROSAT but it was only present at low energies, and so will not affect this measurement. There is therefore very little contamination from other near by sources. We note that J0056 is significantly brighter now than was reported in the RXTE all sky survey (Revnivtsev et al. 2004).

\subsection{IGR J17195-4100}

The peaks in the periodogram of J1719 (1842.4 $\pm 1.5 \mathrm{~s}$ and $2645.0 \pm 4.0 \mathrm{~s})$ are typical for a spin period length in IPs. The small peak at $941 \mathrm{~s}$ is close to being half the 1842.4 period, and therefore may be a first harmonic, however, the significance of this peak is below $3 \sigma$. We note that if the two longer periods above correspond to the spin and beat periods respectively then this implies an orbital period of approximately $1.7 \mathrm{~h}$. The small, almost constant, modulation depth seen in the lightcurves in each energy band is not present among any other confirmed IPs and implies that the modulation is caused by obscuration as opposed to absorption.

The presence of an iron feature at $6.5 \mathrm{keV}$ is a strong indicator of an IP classification. A significantly better spectral fit is obtained from a power law instead of a bremsstrahlung model. The $2-10 \mathrm{keV}$ fluxes obtained from each of the spectral models are also considerably larger than the value reported by Tomsick et al. (2006) in the $0.3-10 \mathrm{keV}$ energy band. This may be indicative of the simplistic single temperature bremsstrahlung model used here; multi-temperature fits are often needed to model the post-shock flow (see e.g. Ezuka \& Ishida 1999). However, the signal to noise and the spectral resolution of the data is such that a complex model may yield non-unique or degenerate results.

There are several X-ray sources near by in the PCA field of view which may contribute to the count rate. We used the ROSAT count rate of each source to estimate an RXTE count rate using the on line tool webPIMMS ${ }^{3}$ (for each source we assume a power law with a photon index of 1.7), we then scaled this value by the response of the PCAs based upon the distance from the source. For this source the contribution is up to $1.1 \mathrm{ct} \mathrm{s}^{-1} \mathrm{PCU}^{-1}$ in the $2-20 \mathrm{keV}$ energy band, i.e. $\$ 20 \%$ of the measured count rate. These extra sources will have the effect of decreasing the percentage modulation depth. Moreover, since it is likely that these sources are softer than the target, the contamination will have a greater effect at lower energies. The modulation depth will therefore be reduced more at lower energies. This could make a decreasing modulation depth with energy look like a constant modulation depth with energy. The spectral fitting is likely to be affected by these other sources and they may be the cause of the poor bremsstrahlung model fit. It is also possible these other sources may skew the model fit in such a way that the calculated flux is then overestimated, this may explain why the flux reported here is larger than the Tomsick et al. (2006) value. We emphasise that this is only an estimate of the contamination; the other sources may differ markedly from the assumed spectral shape.

\subsection{XSS J12270-4859}

J1227 exhibits a structure in the periodogram that indicates it has a period close to 100 cycles day $^{-1}$. This is consistent with being interpreted as a spin period. It shows an approximately constant modulation in each energy band at the $859.57 \pm 0.64 \mathrm{~s}$ period, which implies that the process causing this effect must be a geometrical effect causing obscuration instead of absorption.

The upper limit placed on the equivalent width of a potential iron line is small, and goes against the classification of this as an IP, since all IPs exhibit some kind of iron emission features. We do note however that Masetti et al. (2006) did see significant iron features in their optical spectra. The best spectral fit is obtained from a power law profile, the parameters of which are in good agreement with Revnivtsev et al. (2004). Again we note that a multi-temperature bremsstrahlung fit may be more accurate, but beyond the scope of this study. The count rate has not changed significantly since the measurements of Revnivtsev et al. (2004).

This source also has nearby X-ray sources that may contribute to the count rate. Using the same procedure as outlined above we estimate that they may have contributed up to $0.26 \mathrm{ct} \mathrm{s}^{-1} \mathrm{PCU}^{-1}$ in the $2-20 \mathrm{keV}^{-}$energy band, i.e. $\$ 20 \%$ of the total count rate. It is again possible that these extra sources would alter the modulation depths, and that the spectral fits are also skewed.

${ }^{3}$ http://heasarc.nasa.gov/Tools/w3pimms.html 


\section{Conclusion}

The unambiguous detection of an X-ray spin period of $465.68 \pm$ $0.07 \mathrm{~s}$ in J0056 and its decreasing modulation depth with increasing energy, along with its spectral properties, confirm its inclusion into the IP class. Both J1719 and J1227 clearly exhibit some properties seen in IPs, but not to an extent for us to definitively classify them as such. We do note that it is likely these latter two are IPs, and that their true nature is being masked by the presence of contamination from other sources. X-ray imaging of these sources will definitively decide their fate, allowing their true spectral characteristics to be revealed. All three sources would benefit from long base line optical campaigns to determine their orbital periods and ratify the validity of the periods in J1719 and J1227. If J1227 does turn out to be an IP, then the presence of an X-ray iron feature will have to be reconsidered as a defining characteristic of IPs, since it is not present here.

\section{References}

Barlow, E. J., Knigge, C., Bird, A. J., et al. 2006, MNRAS, 372, 224

Beardmore, A. P., Mukai, K., Norton, A. J., Osborne, J. P., \& Hellier, C. 1998, MNRAS, 297, 337
Bikmaev, I. F., Revnivtsev, M. G., Burenin, R. A., \& Sunyaev, R. A. 2006, Astron. Lett., 32, 588

Bird, A. J., Barlow, E. J., Bassani, L., et al. 2004, ApJ, 607, L33

Bird, A. J., Malizia, A., Bazzano, A., et al. 2007, ApJS, 170, 175

Bradt, H. V., Rothschild, R. E., \& Swank, J. H. 1993, A\&AS, 97, 355

Butters, O. W., Barlow, E. J., Norton, A. J., \& Mukai, K. 2007, A\&A, 475, L29

Dickey, J. M., \& Lockman, F. J. 1990, ARA\&A, 28, 215

Ezuka, H., \& Ishida, M. 1999, ApJS, 120, 277

Hakala, P., Ramsay, G., Wheatley, P., Harlaftis, E. T., \& Papadimitriou, C. 2004, A\&A, 420, 273

Lehto, H. J. 1997, in Applications of time series analysis in astronomy and meteorology, ed. T. Subba Rao, M. B. Priestley, \& O. Lessi (London: Chapman and Hall)

Masetti, N., Morelli, L., Palazzi, E., et al. 2006, A\&A, 459, 21

Norton, A. J., \& Watson, M. G. 1989, MNRAS, 237, 853

Pandey, M., Rao, A. P., Manchanda, R., Durouchoux, P., \& Ishwara-Chandra, C. H. 2006, A\&A, 453, 83

Ramsay, G., Wheatley, P. J., Norton, A. J., Hakala, P., \& Baskill, D. 2008, ArXiv e-prints, 804

Revnivtsev, M., Sazonov, S., Jahoda, K., \& Gilfanov, M. 2004, A\&A, 418, 927

Tomsick, J. A., Chaty, S., Rodriguez, J., et al. 2006, ApJ, 647, 1309

Warner, B. 1995, Cataclysmic variable stars, Cambridge Astrophysics Series (Cambridge, New York: Cambridge University Press) 